\title{
Effect of Smoke Exposure on Chronic Inflammation and P53 Expression in Pelvis Epithelial
}

\author{
Kurnia Penta Seputra, Akmal Fawzi Yusril Umam, Besut Daryanto, Taufiq Nur Budaya, Anita \\ Kenty Watri
}

\begin{abstract}
Many risk factors can cause upper urinary tract carcinoma. Smoking is the most influential risk factor and is associated with the formation of aromatic acids and could induce apoptotic cycle. This research was aimed to examine the effect of cigarete smoke exposure on inflammatory state and p53 expression in renal pelvis epithelial of wistar rats. As many as 25 male rats aged 6-8 weeks were divided into five groups as follows: Control (without treatment); CS-1, CS-2,CS-4, and CS-8 (treated with CS $1 x, 2 x, 4 x$, and $8 x /$ day, respectively). Each exposure was done for 15 minutes for 60 days. histopathological changes were evaluated from HE-stained specimens and immunohistochemistry method was applied to measure p53 expressio neutrophile and lymphocyte count in CS-8 was significantly higher as compared to CS-1 $(p<0.05)$ and control $(p<0.05)$. p53 expression was found in the CS-8 (3 out of 5 subjects had positive p53 expression, with a total of 4-7 cells from 10 hpf). Correlation study showed significant correlation between frequency of cigarette smoking exposure and neutrophile count $(p=0.000 ; r=-0.856) ;$ lymphocyte count $(p=0.000 ; r=0.985)$; and $p 53$ expression $(p=0.000 ; 0.072)$. We concluded that there were significant differences in acute inflammation state, chronic inflammation state and p53 expression among groups. Correlation study showed that frequency of cigarette smoking exposure was positively correlated with lymphocyte count and 553 expression.
\end{abstract}

Keywords: chronic inflammation, cigarette smoke, renal pelvis, p53

\section{INTRODUCTION}

Upper urinary tract carcinoma is a malignancy at urothelium from the kidney dialect to the distal ureter. This cancer has opposing urothelial cancer in the bladder but has different management ${ }^{1}$. This cancer has a prevalence of $5-10 \%$ of all urothelial cancers. It has estimation annual incidence in western countries mostly two cases per 100,000

Revised Manuscript Received on January 2, 2020.

* Correspondence Author

Kurnia Penta Seputra, Departement of Urology, Faculty of Medicine, Brawijaya University, Malang, Indonesia. Email: penta732000@yahoo.com

Akmal Fawzi Yusril Umam, Departement of Urology, Faculty of Medicine, Brawijaya University, Malang, Indonesia.

Besut Daryanto*, Departement of Urology, Faculty of Medicine, Brawijaya University, Malang, Indonesia. Email: besut.daryanto@yahoo.co.id

Taufiq Nur Budaya, Departement of Urology, Faculty of Medicine, Brawijaya University, Malang, Indonesia.

Anita Kenty Wantri, Departement of Pathology Anatomy, Faculty of Medicine, Brawijaya University, Malang, Indonesia. population ${ }^{2}$. Based on data from 2018 cancer statistics in America, 3,820 new cases were obtained with an estimated 960 case fatalities ${ }^{2}$. Evaluation data from the National Cancer Institute's Surveillance, Epidemiology and End Results (SEER) in 1973 to 2005 showed an increase in 9 annual events from 1.88 to 2.06 cases per 100,000 people per year ${ }^{1}$.

Many risk factors can cause urothelial cancer. Smoking is the most influential risk factor and is associated with the formation of aromatic acids which are metabolized into highly carcinogenic N-hydroxylamine. Everyone's susceptibility to the effects of cigarette smoke may be related to genetic changes in the enzymes that neutralize the substance ${ }^{3}$.

One cigarette content, namely nicotine can cause oxidative stress that disrupts the balance of oxidants and antioxidants in blood cells and tissues. Intravenous administration of nicotine in mice can cause urinary mucosal lesions, vascular changes, and parenchymal damage accompanied by infiltration of inflammatory cells. Nicotine increases the production of hydrogen peroxide and superoxide anion which causes disruption in the mitochondrial metabolic chain $^{4}$.

P53 protein is a transcription activator that can induce gene expression in regulating cell cycles, apoptosis, and DNA repair in response to cellular stimuli in the form of hypoxia and DNA damage. Lack of functional p53 protein in cells is known to be related to resistance to chemotherapy, and conversely an increase in p53 levels decreases the occurrence of cancer ${ }^{5}$. P53 protein mutation increases the risk of recurrence and metastasis in cancer. In addition, the p53 protein mutation can be a biomarker for predicting the recurrence of urothelial cancer patients ${ }^{6}$.

In this study, we will examine differences in the frequency of exposure to cigarette smoke to histopathological changes and immunohistological expression of p53 protein in the renal pelvic mucosa of the wistar rat.

\section{MATERIALS AND METHODS}

\section{A. Study Design and Animal Treatment}

This design was designed as true laboratory experimental post-test only controlled group. As many as 25 male rats (aged 6-8 weeks, weighed 200-250 grams) were 
obtained from laboratory of pharmacology, faculty of medicine, brawijaya university. All rats then randomly assigned into five treatment group as follows: (A) control group (without cigarette smoke exposure), (B) Cigarette smoke 1x (CS-1), (C) CS-2, (D) CS-4, and (E) CS-8 (treated with cigarette smoke 1x/day, 2x/day.

4x/day. And 8x/day). Cigarette smoke exposure was done in special chamber connected with smoking pump for 15 minutes/treatment. Cigarette using in this study was obtained from local store (Surya brand, consist of $31 \mathrm{mg}$ tar dan 2,2 nicotine/cigarette).

Of the 25 experimental animals used in this study, 20 experimental animals were exposed to secondhand smoke with a different frequency with variations of exposure 1x/day; 2x/day, 4x/day and 8x/day. After 2 months of exposure to cigarette smoke, differences in rat body weight gain were explained as described in table 1 . For food intake in mice with a frequency of exposure $8 x /$ day, the second month decreased appetite and decreased motion activity. It also found differences in rat fur color with a frequency of exposure to cigarette smoke $4 \mathrm{x} /$ day and $8 \mathrm{x}$ /day experiencing more brownish skin discoloration compared to another group.

All of procedure performed in this study has been approved by ethical committee of medical and health research, faculty of medicine Brawijaya university (400/123/K.3/302/2019).

\section{B. Histology Feature and Chronic Inflammation Score}

Following terminal surgery, renal pelvis was removed, devide into two part, one of them was fixed in $10 \%$ formalin solution. Preparation of histopathological specimen for hematoxylin eosin (HE) staining was conducted as previously described. Briefly, the processes including dehydration, embedded in paraffin block, and sectioning at 5 $\mu \mathrm{m}$ thickness.

Hematoxylin aeosin staining was conducted as previously described. Briefly, specimens were deparaffinized by using xylene, continued with rehydration process using ethanol in reducing manner $(95 \%, 90 \%, 80 \%$, and $70 \%$, respectively). After this process, specimens were stained using hematoxylin and eosin sequentially. At the final step, specimens were dehydrated by using ethanol dan cleared with xylene. The preparation of histopathilogical specimens were conducted in the Laboratory of Pathology Anatomy, Faculty of Medicine, Brawijaya University.

All slide was observed under light microscope (Olympus BX-51) using 400x magnification. Each specimen was observed for 10 random hpf and analyzed for sign of acute inflammation (neutrophil), chronic inflammation (lymphocyte), Proliferation cell, dysplasia and carcinoma.

\section{Measurement of P53 Expression}

Firstly, half part of bladder organ was fixed in the $10 \%$ buffered formalin. After fixation process, the bladder was embedded in paraffin block, sectioned at $5 \mu \mathrm{m}$ thickness by using microtome and put on the polysine adhesion slides. Paraffin-embedded were stained by using immunohistochemistry method.28 Briefly, following deparaffinization using xylene and rehydration using ethanol, specimens were soaked in peroxidase blocking solution and then incubated with prediluted blocking serum. After blocking process, slides were soaked in primary p53 antibody (p53 mouse monoclonal antibody, Santa Cruz Biotechnology, sc-47698, dilution factor 1:250) at room temperature (10 minutes). Slides then washed with PBS and incubated with secondary antibody at room temperature (5 minutes). Slides then washed with PBS and incubated at room temperature (10 minutes). Slides then washed again with PBS and incubated with DAB (diaminobenzidine) chromogen at room temperature (10 minutes). Slides was be mounting by hematoxylin eosin staining. Expression of p53 was defined as brownish appearance in the nucleus of epithelial and stromal cells.

\section{Analysis Data}

Calculation of neutrophil count and lymphocyte count were transformed into ordinal data. Data analysis was conducted by using software SPSS version 24.0 at confidence interval $95 \%$ and use kruskal-wallis to analyze chronic inflammation state and p53 expression, one-way anova to analyze acute inflammation state. Correlations study perform to analyze correlations between frequency of exposure to Inflammation and Expression of p53.

\section{RESULTS}

\section{A. Comparasion of Acute and Chronic Inflamatory Level}

Inflammatory parameters in this study are broadly divided into 2, namely acute and chronic. Acute inflammatory parameters include neutrophil count, edema, congestion, and mucosal erosion. While the parameters of chronic inflammation include lymphocyte count and monocyte count. In this study, acute parameters that have emerged are neutrophils and for chronic inflamation parameters that have appeared, namely lymphocytes. The test carried out to analyze the differences of each variable between the 5 treatment groups on lymphocyte count is the Kruskal-Wallis test. As for the neutrophil count, one-way anova is used. If the $\mathrm{p}$ value $<0.05$, that interpretation is a significant difference between the treatment groups. If the value of $p>$ 0.05 hasn't significant difference between five treatment group.

From the research it was seen that only neutrophil and lymphocyte count variables showed significant differences between the five treatment groups. To find out more which groups were significantly different between the five treatment groups, multiple comparisons were made between the five groups. The results of the comparison can be seen in the Appendix. The value of statistic was a significant difference between neutrophil counts of the control with $4 x$ and $8 \mathrm{x}$ exposure, and between $1 \mathrm{x}$ and $8 \mathrm{x}$ exposure group.

\section{B. Comparision of p53 Expression}

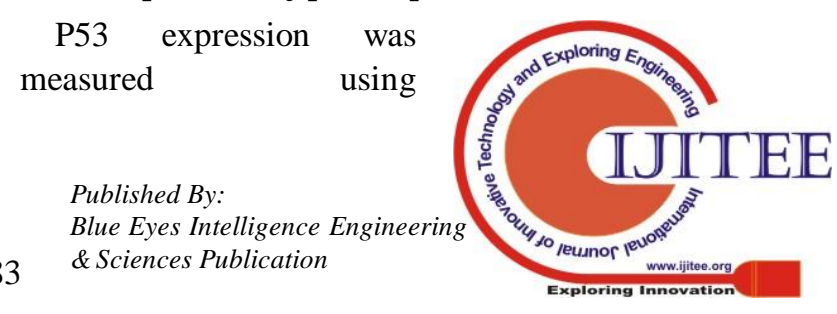


immunohistochemical methods to look semiquantitatively at p53 expression in histology preparations. P53 expression was calculated using a large field of view (40x) by counting the number of brown epithelial or stromal cells in the nucleus. The non-parametric test used Kruskal Wallis, it showed differences in p53 expression between the five treatment groups. If the $\mathrm{p}$ value $<0.05$, it can be concluded that there are significant differences between the five treatment groups.
Conversely, if the value of $\mathrm{p}>0.05$, it means no significant difference between the five treatment groups.

Test results showed that in the control group, 1x exposure, $2 \mathrm{x}$ exposure, and $4 \mathrm{x}$ exposure did not obtain p53 expression. P53 expression was found in the $8 \mathrm{x}$ exposure group (3 out of 5 subjects had positive p53 expression, with a total of 4-7 cells from $10 \mathrm{lpb})$. Kruskal Wallis test results showed significant results on CS-8 $(\mathrm{p}=0.011)$

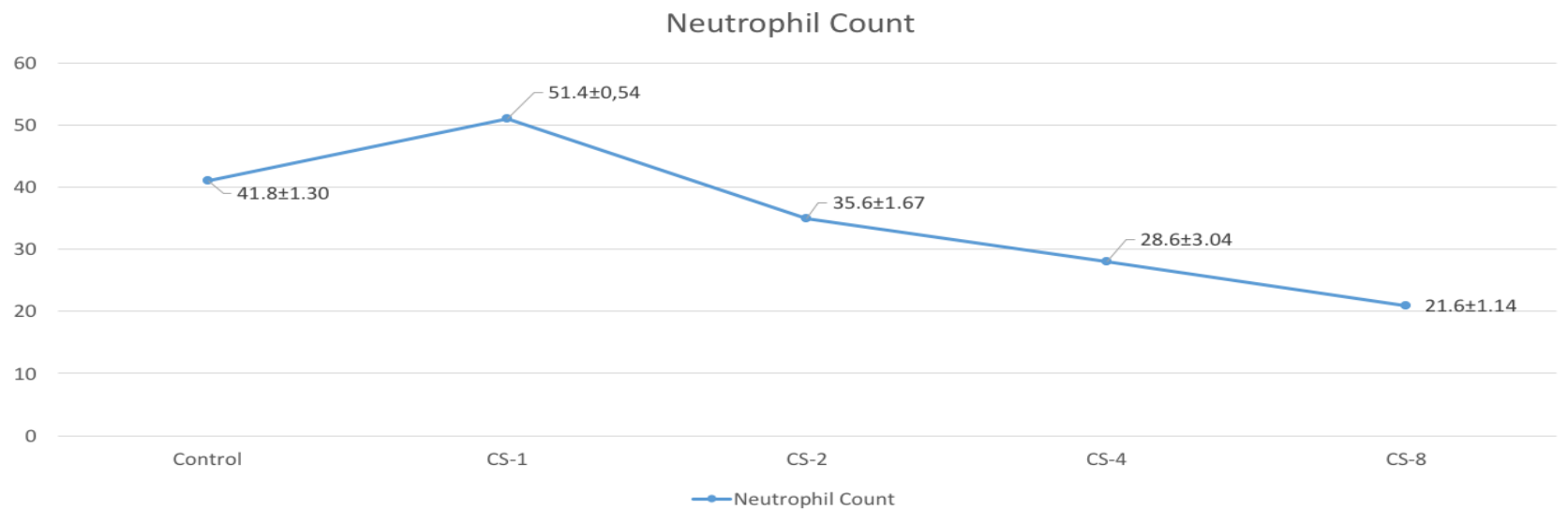

Fig. 1. Neutrophil Count in each group. in acute inflammatory parameters there was a difference in the control treatment with exposure 1x and decreasing in exposure $8 x$

Lymphocyte Count

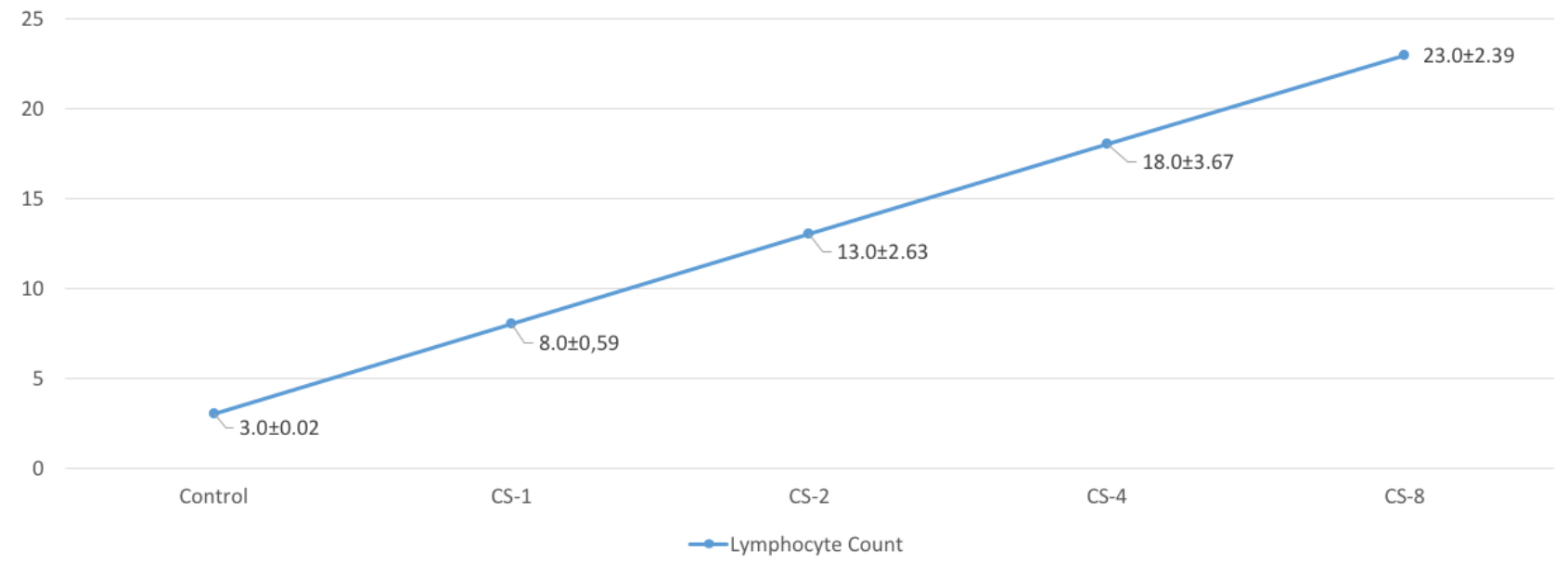

Fig. 2. Lymphocyte Count in each group. in chronic inflammatory parameters there was a difference in the control treatment with exposure $1 x$ and increasing in exposure $8 x$. 


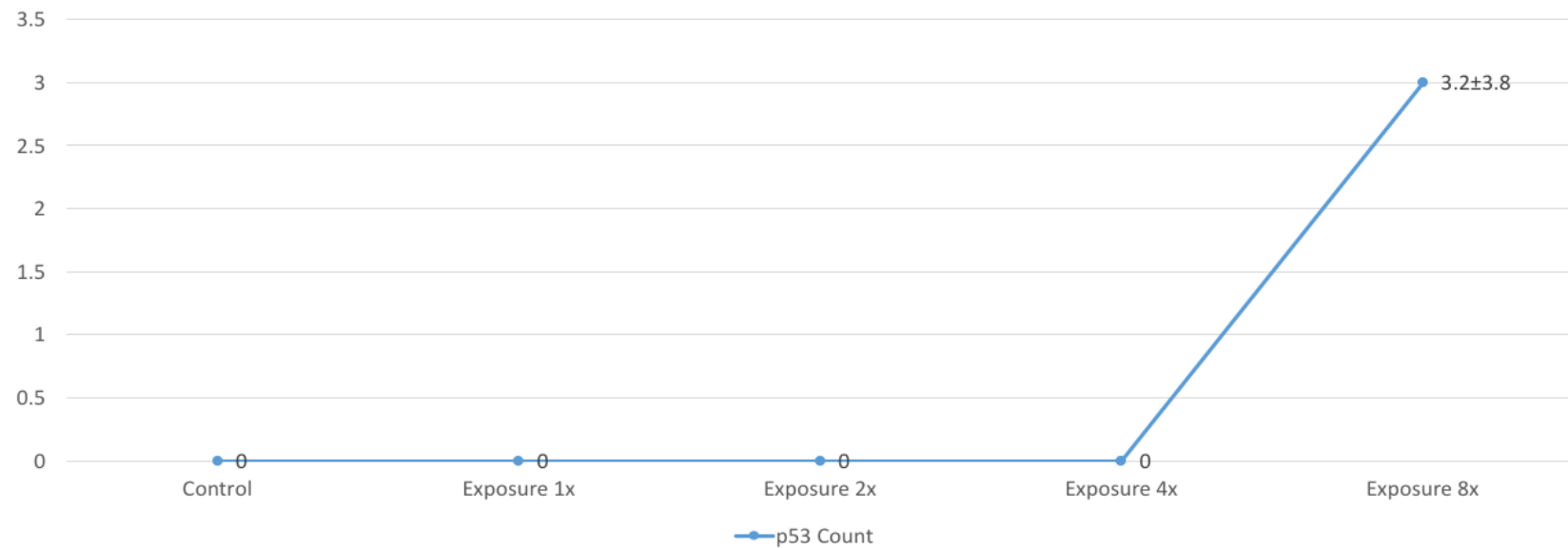

Fig. 3. p53 Count in each group. There is p53 expression in exposure 8x

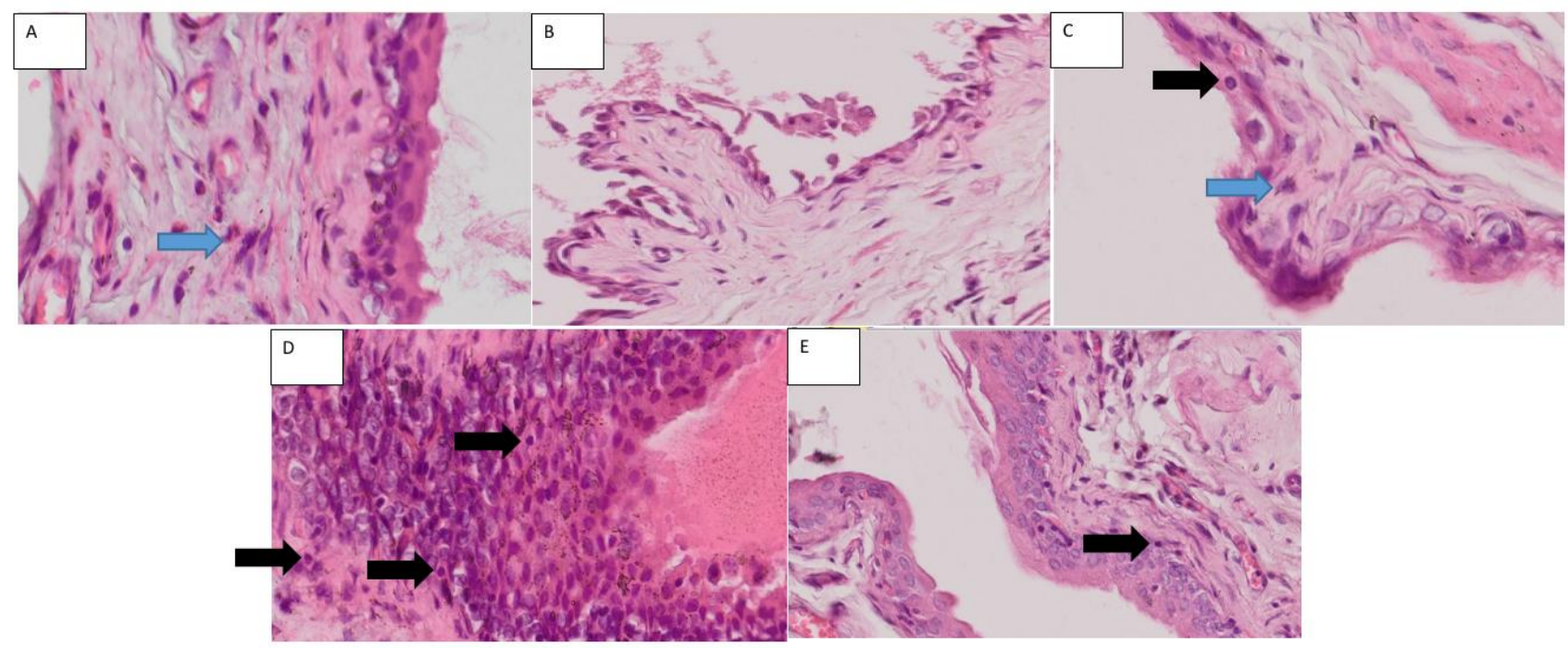

Fig. 4. The results of hematoxylin eosin staining to assess the histopathological picture through the Olympus BX-51 microscope 400x magnification (10 large field of view) in each treatment group: (A) control, (B) exposure 1x (magnification 200x) (C) exposure 2x, (C) exposure D) 4x exposure, and (E) 8x exposure. Some signs indicate neutrophils (blue circles), and lymphocytes (black circles).

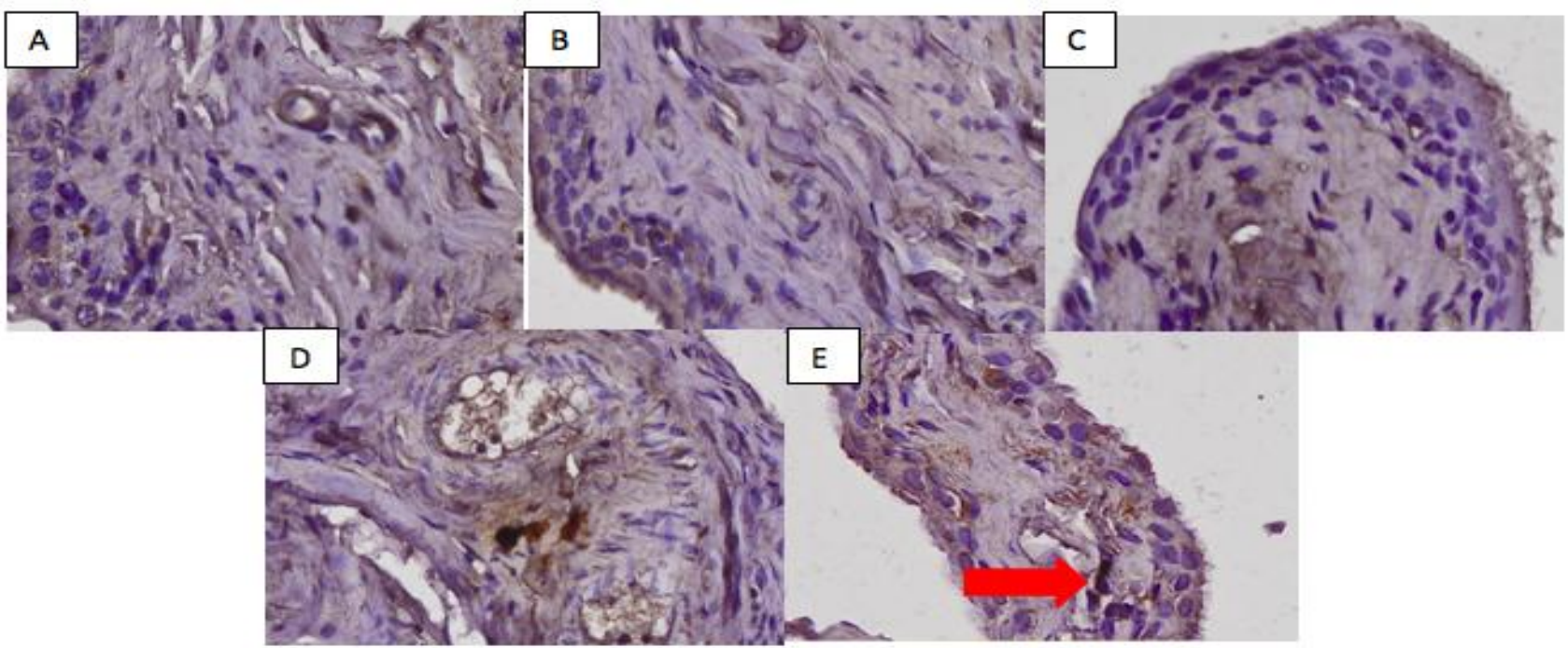

Fig. 5. Immunohistochemical staining results to assess p53 expression through an Olympus BX-51 microscope with

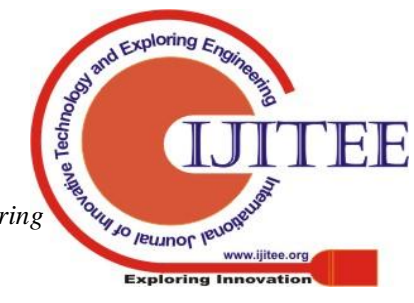


400x magnification (10 large visual fields) in each treatment group: (A) control, (B) exposure 1x, (C) exposure 2x, (D) exposure 4x, and (E) 8x exposure. Positive p53 expression was found in the 8x exposure group (red arrow).

\section{Correlation of cigarette smoke exposure frequency with inflammation and p53 expression}

The next test is to determine the correlation between the frequency of cigarette smoke exposure with each variable. Correlation test is done by the Spearman test (non-parameteric). From the results of the correlation test will get the value of $p$ and $r$. If the value of $p<0.05$, it can be interpreted that has correlation between frequency of exposure and the variable of exposure, whereas if the value of $p>0.05$, the interpretation shows that there is no correlation. The value of $r$ indicates the strength of the correlation. From this study, the frequency of cigarette smoke exposure was negatively correlated with neutrophils whereas lymphocytes and p53 were positively correlated.

TABEL 1. CORRELATION OF CIGARETTE SMOKE EXPOSURE FREQUENCY WITH INFLAMMATION AND P53 EXPRESSION

\begin{tabular}{|c|c|c|c|}
\hline Parameter & Variabel & P Value & R Value \\
\hline Inflammation & $\begin{array}{c}\text { Neutrophil } \\
\text { count }\end{array}$ & 0.000 & -0.856 \\
\hline & $\begin{array}{c}\text { Lymphocyte } \\
\text { count }\end{array}$ & 0.000 & 0.985 \\
\hline p53 Expression & P53 count & 0.000 & 0.702 \\
\hline
\end{tabular}

\section{DISCUSSION}

\section{A. Correlation of cigarette smoke exposure and inflammation}

In this study, statistical tests have been carried out to see the differences in each inflammatory variable in each treatment group. The results had a significant correlation between the frequency of cigarette smoke exposure with chronic inflammatory variables including lymphocyte counts. Conversely, the frequency of exposure to cigarette smoke is negatively correlated with the acute inflammatory parameter, neutrophils. This is related to the long exposure to cigarette smoke for 8 weeks included in the chronic category.

The effect of exposure to cigarette smoke is mediated by nicotine and other toxic substances contained. This substance when entered into the biological system will induce the development of oxygen free radicals or reactive oxygen species (ROS) and disrupt the function of mitochondria so that it will induce cell apoptosis7. Previous studies have reported that smoking can increase the chronic inflammatory status characterized by increased kappa b (NF- kB) nuclear signaling factor in the lung which can induce the occurrence of chronic obstructive pulmonary disease and lung cancer8. Activation of NF- $\kappa \mathrm{B}$ will induce transinflammatory cytokine transcription and suppression of anti-inflammatory gene transcription.

Lymphocytes and neutrophils have also been investigated as predictor factors for the prognosis urothelial carcinoma using clinical parameters of the neutrophil-lymphocyte ratio. The results show that the neutrophil-lymphocyte ratio is significantly related to prognosis and is associated with tumor stage, lyphovascular invasion and also level of tumor ${ }^{9}$.

The effect of smoking on epithelial damage to the upper urinary tract has also been studied. From the results of multivariate analysis conducted miyata and his team showed that ex-smoking correlates with lymphatic vessel density (LVD) and intratumoral lymphatic vessels (iLV). In survival analysis, LVD, the presence of iLV, expression of cyclooxygenase (COX)-2 and matrix metalloproteinase (MMP)-9 were identified as metastasis predicting factors after surgery. In conclusion, lymphangiogenesis and expression levels of COX-2 and MMP-9 were found to be associated with smoking patients with upper urinary tract malignancy ${ }^{10}$.

Several previous studies have reported that increased lymphovascular invasion and lymphangiogenesis are important stages in the spread of cancer cells in the upper urinary tract of malignancy. However, information about the influence of smoking status on lymph angiogenesis with the upper urinary tract malignancy is still not very much. Lung cancer had high association with smoking exposure, that of expression of interleukin-17 (IL-17), It was hypothesized to have an important role in metastasis by increasing lymph angiogenesis. Additionally, although lymph angiogenesis and COX-2 or MMP-9 expression had relationship in the upper urinary tract malignancy is not fully understood, that was reported the two molecules have been reported to stimulate lymphangiogenesis in other types of cancer. Therefore, it can be said that smoking induces the spread of cancer cells by stimulating lymphangiogenesis through increased regulation of COX-2 pathway and cytokine MMP-9 in patients with urothelial carcinoma of the upper urinary $\operatorname{tract}^{10}$.

In addition, smoking is also associated with the pathological characteristics of urothelial carcinoma in the upper urinary tract affecting worse prognostic factors for recurrence and metastasis after surgery. Several other studies have proven that smoking intensity is a significant predictor of prognosis and survival in patients with urothelial urinary tract carcinoma ${ }^{11,12}$.

\section{B. Correlation of cigarette smoke exposure and p53}

This research shows that exposure to cigarette smoke within 8 weeks can affect the expression of p53. Cells with p53 expression began to appear to be increased at $8 x$ exposure and were significantly different compared to all treatment groups. It has been previously known that p53 is a tumor-suppressor that is encoded by the p53 gene (p53). P53 molecule is a transcription factor with the main function as a regulator of the cell cycle and often mutates in various cases of human malignancy, which is about $50 \%$ of all malignancies in humans. p53 was discovered in the late 1970 s in the form of a cellular 53-kd nuclear 
phosphoprotein bound to the SV40 DNA virus. This protein in addition to influencing cell cycle control, also plays a role in repairing DNA damage and DNA synthesis, cell differentiation, and cell death programs (apoptosis) ${ }^{13}$.

BCL-2 family and tumor suppressors such as the P53 protein are many characteristics of types cancers, including bladder cancer. This decrease gives cancer cells the ability to resist apoptosis, thereby supporting cell survival. Tobacco consumption is documented that risk factor for developing urinary tract cancer, but it wasn't explained clearly about carcinogenic mechanism and molecular pathways. Many studies have focused on aromatic amines such as 4-aminobiphenyl (ABP). 4-aminobiphenyl found in cigarette smoke and some industrial chemicals. One potential mechanism by which aromatic amines caused carcinogenesis is by forming DNA mutation that produces transitional mutations $^{14}$.

Cigarette smoke containing nitrosamine has been known to be a specific carcinogen in urothelial. It is known that N-butyl-N- (4-hydroxybutyl) nitrosamine (BBN) changes the expression of GSTM1, GSTO1 and NQO1 proteins in urotelium of mice after 20 weeks of treatment. GSTM1, an antioxidant enzyme associated with the development of urothelial cancer also has decreased expression at both protein and mRNA levels and this is mediated in part by DNA methylation ${ }^{15}$.

In this research, IHC p53 examined the prognostic role of p53 expression in 83 patients with in the upper urinary tract carcinoma. Our result that p53 overexpression was significantly associated with tumor aggressiveness and prognostic patients. Tumor expression, grade, and p53 were all significantly related to results in univariate analysis. However, tumor level was not a predictor in multivariable analysis whereas p53 expression status remained significant $^{16}$.

In Japanese studies $(n=66)$ and Europe $(n=53)$, p53 was a predictor factor in univariable analysis but did not appear as an independent prognostic factor after adjusting for clinical and pathological characteristics. IHC study examined the expression of p53 in 112 UTUC patients and found high p53 expression was a bad predictor progression of disease (hazard ratio $[\mathrm{HR}]=3.74, \mathrm{p}=0.025$ ) and survival $(\mathrm{HR}=5.87, \mathrm{p}=0.030)^{17}$.

\section{CONCLUSION}

There was a significant difference in acute, chronic inflammation state and p53 expression among groups. Correlation study showed that frequency of cigarette smoking exposure was positively correlated with chronic inflammation and p53 exposure.

\section{ACKNOWLEDGMENT}

We thank Mochammad Abuhari, Lasmijan, and Heni Triwahyuni for direct technical assistance during the study.

\section{REFERENCES}

1. Smith AK, Matin SF, Jarrett TW. Urothelial Tumors of Upper Urinary Tract and Ureter. Campbell Walsh Urology 2016: 1365-1401.

2. Siegel, R.L., et al. Cancer statistics, 2017. CA Cancer J Clin, 2017. 66: 7.

3. Roupret M, Hupertan V, Seisen T, et al. Prediction of cancer specific survival after radical nephroureterectomy for upper tract urothelial carcinoma: development of an optimized postoperative nomogram using decision curve analysis. J Urol 2013;189:1662-9.

4. Pekmez,H. Ogeturk, M., Ozyurt, H., Sonmez, M. F., Colakoglu, N., \& Kus, I. (2010). Ameliorative effect of caffeic acid phenethyl ester on histopathological and biochemical changes induced by cigarette smoke in rat kidney. Toxicology and Industrial Health, 26(3), 175-182.

5. Aubrey, B. J., Strasser, A., \& Kelly, G. L. (2016). Tumor-Suppressor Functions of the TP53 Pathway. Cold Spring Harbor Perspectives in Medicine, 6(5), a026062.

6. Chen CH, Dickman KG, Huang CY, Shun, CT, Tai HC, Huang, KH, et al Recurrence pattern and TP53 mutation in upper urinary tract urothelial $\begin{array}{lll}\text { carcinomar } & \text { Oncotarget. 2016;7(29):45225-45236 }\end{array}$ doi:10.18632/oncotarget.9904.

7. Arany, I. Clark, JS. Faisal, A. p66SHC-Mediated Mitochondrial Dysfunction in Renal Proximal Tubule Cells During Oxidative Injury. 2010. AJP Renal Physiology 298(5):F1214-21

8. Zaynagetdinov R., Sherrill T. P., Gleaves L. A., Hunt P., Han W., McLoed A. G., Saxon J. A. Tanjore H., Gulleman P. M., Young L. R., Blackwell T. S. Chroni c NF- $\kappa$ B activation links COPD and lung cancer through generation of an immunosuppressive microenvironment in the lungs. Oncotarget. 2015; 7: 5470-5482.

9. Li X., Ma X., Tang L., Wang B., Chen L., Zhang F., Zhang X. Prognostic value of neutrophil-to-lymphocyte ratio in urothelial carcinoma of the upper urinary tract and bladder: a systematic review and meta-analysis. Oncotarget. 2017; 8: 62681-62692.

10. Miyata Y, Mitsunari K, Akihiro A, Watanabe SI, Mochizuki Y, Sakai H. Smoking-induced changes in cancer-related factors in patients with upper tract urothelial cancer. Molecular and Clinical Oncology. 2015;3(2):287-294.

11. Ehdaie B, Furberg H, Zabor EC, et al: Impact of smoking status at diagnosis on disease recurrence and death in upper tract urothelial carcinoma. BJU Int 111: 589-595, 2013.

12. Hagiwara M, Kikuchi E, Tanaka N, et al: Impact of smoking status on bladder tumor recurrence after radical nephroure- terectomy for upper tract urothelial carcinoma. J Urol 189: 2062-2068, 2013.

13. Lefevre PJ. Pharmacology of minor alkaloids of tobacco [French]. Journée de la Dépendance Tabagique 2011; 65(40):2424-32

14. Jung, I. Messing, E. Molecular Mechanism and Pathways in Bladder Cancer Development and Progression. 2010. Cancer Control July/August, Vol. 7 No. 4. 325-334

15. Wang NP, Lim PS, Chen TT, Thien LM, Wang TH, and Hsu WM. Smoking is associated with alterations of blood thiol-group related antioxidants in patients on hemodialysis. 2012Nephron 92: 772-779

16. Li, Q., Bagrodia, A., Cha, E. K., \& Coleman, J. A. (2016). Prognostic Genetic Signatures in Upper Tract Urothelial Carcinoma. Current Urology Reports, 17(2).

17. Zigeuner $\mathrm{R}$ et al. Prognostic impact of $\mathrm{p} 63$ and $\mathrm{p} 53$ expression in upper urinary tract transitional cell carcinoma. Urology. 2014;63(6):1079

\section{AUTHORS PROFILE}

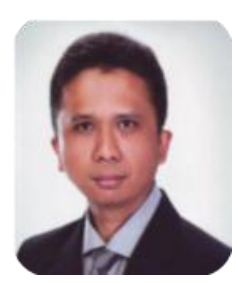

dr. Kurnia Penta Seputra Sp. U (K) has completed his urologist degree from Airlangga University. He is currently working as urology staff in Urology Department, consultant in Uro-oncology, Brawijaya University, Malang. He has published more than 20 research paper in various national and international journal.

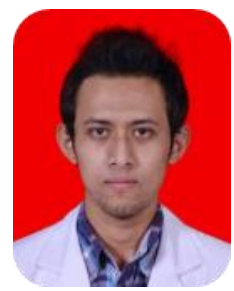

dr. Akmal Fawzi Yusril Umam has completed his medical degree in Faculty of Medicine, Brawijaya University. He is currently as urology resident in Brawijaya University, Malang. 


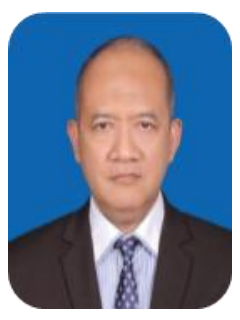

Dr. dr. Besut Daryanto Sp. B, Sp. U (K) has completed his general surgeon from Diponegoro University, and urologist degree from Airlangga University. He has completed his doctoral from Brawijaya University. He is currently working as a Head of Urology Residency Program Medical School of Brawijaya University, Malang. He has published 20 research paper in International Journal.

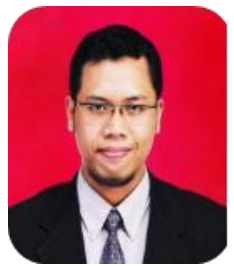

dr. Taufiq Nur Budaya Sp. U has completed his urologist degree from Airlangga University, He is currently working as urology staff at Urology Department, Brawijaya University, Malang. He has released 2 books about BPH and Malignancy in Urinary Tract.

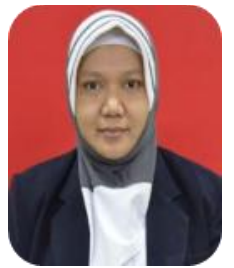

dr. Kenty Wantri Anita Sp. PA has completed his pathology anatomist degree from Diponegoro University. She is currently working as pathology anatomy staff in Pathology Anatomy Department, Brawijaya University, Malang. 